\title{
SOME LIMIT THEOREMS FOR CONTINUOUS-STATE BRANCHING PROCESSES
}

\author{
ANTHONY G. PAKES \\ (Received 17 March 1986; revised 24 June 1986) \\ Communicated by T. C. Brown
}

\begin{abstract}
The most general continuous time and state branching (C.B.) process $\left(X_{t}\right)$ can be constructed as a certain random time transformation of a spectrally positive Lévy process. When the generating process is compound Poisson with a superimposed negative linear drift and the C.B. process is not supercritical, then there is a random time $T$ such that $X_{t+T}=e^{-c t} X_{T}$ where $c>0$ is the drift parameter. Thus $T$ is the last epoch of random variation.

The paper explores a similar phenomenon for the discrete time case and it presents some conditional limit theorems related to the last epoch of random variation.

A secondary objective is to present some limit theorems for the C.B. process analagous to known results for the discrete time case.
\end{abstract}

1980 Mathematics subject classification (Amer. Math. Soc.): $60 \mathrm{~J} 80$.

\section{Introduction}

We begin by considering a Jiřina process (J.P.) $\left(Z_{n}: n \in \mathbb{N}_{+}\right)$which is a Markov chain with state space $[0, \infty)$ and whose transition kernel is defined by

$$
E\left(\exp \left(-s Z_{n+1}\right) \mid Z_{n}\right)=\exp \left(-Z_{n} h(s)\right)
$$

where $h$ is the cumulant generating fucntion (c.g.f.) of a necessarily infinitely divisible distribution function (D.F.) $F$. This process can be interpreted as a branching process where $Z_{n}$ measures the quantity of "population" in the $n$th generation and $F$ is the D.F. of the quantity of progeny due to a unit quantity of "parent". The definition incorporates the basic property of independence of lines of descent of disjoint subsets of the population. We set $Z_{0}=z>0$.

() 1988 Australian Mathematical Society $0263-6115 / 88 \$ A 2.00+0.00$ 
In this paper we consider the case where $F$ is non-degenerate, $m=\int_{0}^{\infty} x d F(x)$ $\leqslant 1$ and $r=\lim _{s \rightarrow \infty} h(s) / s$, the first point of increase of $F$, satisfies $0<r<1$. In this case $Z_{n} \rightarrow 0$ a.s. but $P\left(Z_{n}>0 \forall n\right)=1$ and there is a sequence of constants satisfying $c_{n} \uparrow \infty, c_{n+1} / c_{n} \rightarrow r^{-1}$ and $c_{n} Z_{n} \rightarrow Z$ a.s. where $Z$ has a proper non-degenerate D.F. Convergence in distribution was first shown by Seneta and Vere-Jones [12] and the extensions to a.s. convergence was independently observed by others-see Pakes [7] for references. Kallenberg [4] also obtained this limit theorem, apparently in ignorance of the work in [12].

Pakes [7] investigated the rate of convergence of $\left(c_{n} Z_{N}\right)$ to $Z$. His results require some extra assumptions on $F$. The function $k(s)=h(s)-r s$ is a c.g.f. having the canonical representation

$$
k(s)=\int_{0+}^{\infty}\left(1-e^{-s x}\right) n(d x)
$$

where $n$ is a Lévy measure, that is, it is a positive measure on $(0, \infty)$ satisfying $\int_{0}^{\infty}(1 \wedge x) n(d x)<\infty$. Observe that $k(s) / s \rightarrow 0$ as $s \rightarrow \infty$. The conditions used by Pakes ensure that for each $\varepsilon>0$,

$$
\int_{\varepsilon}^{\infty} s^{-2} k(s) d s<\infty
$$

and in this case [12] $c_{n} \sim$ const. $r^{-n}(n \rightarrow \infty)$. In this paper we shall use $c_{n}=r^{-n}$ whenever (1.1) is in force. Assuming this is the case, define

$$
W_{n}=Z-r^{-n} Z_{n} \text {. }
$$

The limit $Z$ has a c.g.f. $\gamma(s)$ which, up to scale factors, is the unique solution of

$$
h(\gamma(s))=\gamma(r s)
$$

and $\lim _{s \rightarrow \infty} \gamma(s) / s=1$. Let $a(s)=\gamma(s)-s$. Pakes [7] obtained the following result on the behaviour of $\left(W_{n}\right)$.

THEOREM 1.1. Let (1.1) hold. Then

(i) $P\left(W_{n} \geqslant 0\right)=1$.

Suppose $k(s)=s^{\delta} L(s)$ where $0 \leqslant \delta \leqslant 1, L$ is slowly varying (S.V.) at infinity and $\int_{\varepsilon}^{\infty} s^{-1} L(s) d s<\infty$ if $\delta=1$.

(ii) If $0<\delta \leqslant 1$ there is a strictly increasing $\delta^{-1}$-varying function $g$ such that

$$
r^{n} g\left(r^{-n}\right) W_{n} \stackrel{\mathscr{D}}{\rightarrow} S Z^{1 / \delta}
$$

where $S$ is stable with c.g.f. $s^{\delta}$ and independent of $Z$. In addition

$$
r^{n} g\left(Z_{n}^{-1}\right) W_{n} \stackrel{\mathscr{D}}{\rightarrow} S .
$$

(iii) If $\delta=0$ and $L(s) \rightarrow \infty$, that is, $n((0, \infty))=\infty$, then

$$
\gamma\left(r^{n} a\left(r^{-n} W_{n}^{-1}\right)\right) \stackrel{\mathscr{D}}{\rightarrow} \Delta
$$

where $P(\Delta>x)=e^{-x}$. 
Some remarks are in order about the original statement of this result. First, the exponent $1 / \varepsilon$ on the right hand side of $(1.3)$ was omitted. The limiting c.g.f. was shown to be $\gamma\left(s^{e}\right)$ but this was incorrectly interpreted. Secondly, the condition $n((0, \infty))=\infty$ was omitted in the statement of (iii), but was certainly used in its proof. Thus in both parts (ii) and (iii) it is assumed that $n((0, \infty))=\infty$. One of our goals is to investigate the case $\lambda=n((0, \infty))<\infty$, where, as we shall see, a.s. the sequence $\left(W_{n}\right)$ has finitely many positive terms and hence there can be no non-trivial limit theorem for $\left(W_{n}\right)$.

Corresponding to the c.g.f. $h$ is a right continuous subordinator $(T(t))$ :

$$
E(\exp (-s T(t))=\exp (-\operatorname{th}(s)) \text {. }
$$

If $\lambda=\infty$ then almost all sample paths of $T(\cdot)$ have a set of jump points which is dense in $\mathbf{R}_{+}$, but if $\lambda<\infty$ then $\Gamma(t)=T(t)-r t$ is a compound Poisson process with rate $\lambda$ and jump size D.F. $\lambda^{-1} n((0, x])$. Thus Theorem 1.1 excludes the compound Poisson case. In a sense to be made clear below, when $\lambda<\infty$ there is a random time $\nu$ after which the population size declines deterministically and geometrically fast. This behaviour is exactly analagous to extinction in a GaltonWatson process and a corresponding theory can be developed by seeking conditional limit theroems for $Z_{n}$, or $W_{n}$, given $v>n$. We shall do this in Section 3. The existence of $\nu$ and associated distribution results will be the subject of Section 2.

The existence of the behaviour outlined above is suggested by that of the continuous time and state branching (C.B.) process $\left(X_{t}: t \geqslant 0\right)$ with no Gaussian component. This can be constructed as a randomly time changed version of the first positive excursion of a right-continuous spectrally positive Lévy process $\left(L_{t}: t \geqslant 0\right)$ where $L_{0}=z>0$,

$$
E\left[\exp \left(-s\left(L_{t}-L_{0}\right)\right)\right]=\exp \left[t\left(c s-\int_{0+}^{\infty}\left(1-e^{-s x}\right) \Pi(d x)\right)\right],
$$

$c>0$ and $\Pi$ is a Lévy measure with $\Pi(\{\infty\})=0$; see [8] for references. When $a=\Pi((0, \infty))<\infty$ this construction can be explicitly carried out $[8]$ and it shows that almost all sample paths consist of segments which decrease exponentially fast, $d X_{t}=-c X_{t} d t$, and these segments are separated by random increments occuring at random times. Let $J_{0}=0$ and $J_{1}, J_{2}, \ldots$ denote the successive jump times of $\left(L_{t}\right)$. When $-\infty<D=-c+\int_{0}^{\infty} x \Pi(d x) \leqslant 0$ there is a last jump time $J$ prior to $\inf \left\{t: L_{t}=0\right\}$. The random time change sends $J$ to a time $T<\infty$ (a.s.) and for $t \geqslant T, X_{t}=X_{T} e^{-c(t-T)}$. Then, a.s. as $t \rightarrow \infty$,

$$
e^{c t} X_{t} \rightarrow X=X_{T} e^{c T}
$$

and $e^{c t} X_{t}=X$ if $t \geqslant T$. The discrete skeleton at scale $h,\left(X_{n h}: n \in N_{+}\right)$is a J.P. having the sample path behaviour further elucidated in the next section. 
Pakes and Trajstman [8, Theorems 4.4 and 4.5] obtained conditional limit theorems for $\left(X_{t}\right)$ and $\left(e^{c t} X_{t}-X\right)$ conditional on the even $T>t$. Theorems 3.1, $3.2,3.4,3.5$ are the corresponding results for the J.P. In Section 4 we complete the correspondence by obtaining a version of Theorem 1.1 for C.B. processes in the case where $a=\infty$.

When $1<m<\infty$ for the J.P. it was shown by Seneta and Vere-Jones [12], and rather later by Kallenberg [4], that there exists a sequence $\left(d_{n}\right)$ such that $d_{n} \uparrow \infty$, $d_{n+1} / d_{n} \rightarrow m$ and $\left(Z_{n} / d_{n}\right)$ converges a.s. to a proper non-degenerate random variable. Kallenberg showed also that the limiting D.F. is absolutely continuous on $(0, \infty)$ and may have an atom at the origin. When $1<m \leqslant \infty$ Pakes proved it was possible to obtain a weak limit result for the un-normalized process $\left(Z_{n}\right)$ by diluting the initial population quantity. In Section 4 we will extend this result to the C.B. process where we can obtain a slightly more explicit representation for the c.g.f. of the limiting distribution.

Returning to the C.B. process with $D \leqslant 0$ and $a<\infty$, it is possible to decompose the sample path integral $\mathscr{I}=\int_{0}^{\infty} X_{t} d t$ into two components, viz., $\int_{0}^{T} X_{t} d t$ and $\int_{T}^{\infty} X_{t} d t$. The second component, $\mathscr{I}_{R}$, is the contribution to $\mathscr{I}$ by that portion of the sample path following the cessation of random variation and clearly $\mathscr{I}_{R}=c^{-1} X_{T}$. Following the main theme of this paper we will, in Section 5, investigate the conditional distribution of $X_{T}$ given $T>t$ and obtain a limit theorem without extra assumptions. When $D=0$ we obtain an explicit limiting Laplace-Stieltjes transform. The joint behaviour of $\mathscr{I}^{-} \mathscr{I}_{R}$ and $\mathscr{I}_{R}$ is also of interest but we will not pursue this here. In Section 5 we will also look at the corresponding question for the J. P., that is, we look at the total quantity of population following $\nu$.

It is interesting to compare these results with a similar one for the simple Galton-Watson process $\left(U_{n}\right)$. Let $M=E_{1}\left(U_{1}\right) \leqslant 1$ and $N$ be the extinction time. We shall show that the distribution of $U_{n-1}$, given $N>n$, tends as $n \rightarrow \infty$ to one which is closely related to the $M^{-1}$-invariant measure of $\left(U_{n}\right)$. There seems to be no similar representation for the limit distributions in the continuous-state case. Indeed, under our assumptions these processes have no such invariant measures.

There is some duplication of notation, but not within sections or models.

\section{Construction of the J.P.}

Let $(T(t): t \geqslant 0)$ be a subordinator as defined in Section 1 satisfying (1.5) and let $Z_{0}=z$. Kallenberg [4] constructs the J.P. by defining $S_{-1}=0$ and assuming that $Z_{0}, \ldots, Z_{n}$ have been determined he sets $S_{n}=\sum_{j=0}^{n} Z_{j}$ and $Z_{n+1}=T\left(S_{n}\right)-$ $T\left(S_{n-1}\right)$. Then $S_{n}=z+T\left(S_{n-1}\right)$ and the $S_{n}$ are stopping times for $(T(t))$. 
When $\lambda<\infty,(T(t))$ is a compound Poisson process $\left(\Gamma_{t}\right)$ with a super-imposed linear drift of rate $r$. Denote the successive jump times of $\left(\Gamma_{t}\right)$ by $\left(\tau_{n}: n \geqslant 1\right)$, a sequence defining a Poisson process with rate $\lambda$. If $S_{n-1}$ and $S_{n}$ are not separated by a jump time then

$$
Z_{n+1}=T\left(S_{n}\right)-T\left(S_{n-1}\right)=r\left(S_{n}-S_{n-1}\right)=r Z_{n} .
$$

If $S_{n}$ and $S_{n+1}$ also are not separated by a jump time then $Z_{n+2}=r Z_{n+1}=r^{2} Z_{n}$, and so on. Thus until $\left(S_{n+j}: j \geqslant 0\right)$ exceeds a jump time the J.P. sample paths decrease geometrically fast with ratio $r$.

If $S_{n-1}$ and $S_{n}$ are separated by at least one jump time and the associated total increment of $\left(\Gamma_{t}\right)$ is $I$ then

$$
Z_{n+1}=T\left(S_{n}\right)-T\left(S_{n-1}\right)=I+r\left(S_{n}-S_{n-1}\right)=I+r Z_{n} .
$$

Now a.s. $S_{n} \uparrow S<\infty$ and (see [4, page 26]).

$$
S=\inf \{t: T(t-) \leqslant t-z\}=\inf \left\{t: z+\Gamma_{t-} \leqslant(1-r) t\right\} .
$$

Graphical considerations show that a.s. there is a random index $\nu$ such that $\left\{S_{v-2}, S_{v-1}\right\}$ is the last separated pair-define $\nu=0$ if there is no separated pair. More formally define $\nu=\max \left\{n \geqslant 1: Z_{n} \neq r Z_{n-1}\right\}$ where $\max \varnothing=0$. This definition implies that $Z_{\nu+n}=Z_{\nu} r^{n}(n \geqslant 0)$. In particular $Z=r^{-\nu} Z_{\nu}$ and $W_{\nu+n}$ $=0$ a.s. Consequently random variation ceases after the epoch $\nu$. In the following theorem which should be compared with Theorem 4.3 in [8], we obtain results giving the joint distribution of $Z_{n}$ and $\nu$. Let $P_{z}(\cdot)=P\left(\cdot \mid Z_{0}=z\right)$.

THEOREM 2.1 Let $\rho=\lambda /(1-r)$ and $m \leqslant 1$. Then

$$
P_{z}(\nu \leqslant n)=\exp \left(-z h_{n}(\rho)\right)
$$

and

$$
E_{x}\left(e^{-s Z_{n}} ; \nu>n\right)=\exp \left(-z h_{n}(s)\right)-\exp \left(-z h_{n}(s+\rho)\right)
$$

where $h_{n}$ is the $n$-fold iterate of $h$ and $h_{0}(s)=s$. In particular $P_{z}(\nu<\infty)=1$.

Proof. The event $\{\nu=0\}$ occurs if and only if $Z_{n+1}=r Z_{n}(n \geqslant 0)$ if and only if $\tau_{1}>S=z \sum_{n=0}^{\infty} r^{n}=z /(1-r)$. Thus

$$
P_{z}(\nu=0)=P\left(\tau_{1}>z /(1-r)\right)=e^{-\rho z},
$$

which agrees with (2.1). If $n \geqslant 1$,

$$
\{\nu \leqslant n\}=\bigcap_{j=n}^{\infty}\left\{Z_{j+1}=r Z_{j}\right\}=\left\{\text { no jump in }\left[S_{n}, S_{n}+Z_{n} /(1-r)\right]\right\} \text {. }
$$

Since the $S_{n}$ are stopping times, we have, if $\mathscr{F}_{n}=\sigma\left(Z_{0}, \ldots, Z_{n}\right)$,

$$
\begin{aligned}
P_{z}(\nu \leqslant n) & =E_{z}\left(P\left(\text { no jump in }\left[S_{n}, S_{n}+Z_{n} /(1-r)\right] \mid \mathscr{F}_{n}\right)\right) \\
& =E_{z}\left(e^{-\rho Z_{n}}\right)=e^{-z h_{n}(\rho)} .
\end{aligned}
$$


To obtain (2.2) observe that

$$
\begin{aligned}
E_{z}\left(e^{-s Z_{n}} ; \nu>n\right) & =E_{z}\left(e^{\left.-s Z_{n} P\left(\nu>n \mid \mathscr{F}_{n}\right)\right)}\right. \\
& =E_{z}\left(e^{\left.-s Z_{n} P_{Z_{n}}(\nu>0)\right)}\right. \\
& =E_{z}\left[e^{-s Z_{n}}-e^{-(s+\rho) Z_{n}}\right] .
\end{aligned}
$$

That $\nu$ is non-defective follows because $\lim _{n \rightarrow \infty} h_{n}(s)=0$.

\section{Conditional limit theorems}

We begin by looking at limit theorems for $Z_{n}$ given that $\nu>n$, that is, we condition on the event that random variation has not ceased. The final remark in the proof of Theorem 2.1 yields

$$
E_{z}\left(e^{-s Z_{n}} \mid \nu>n\right) \sim \frac{h_{n}(s+\rho)-h_{n}(s)}{h_{n}(\rho)} \quad(n \rightarrow \infty) .
$$

When $m<1$ Seneta and Vere-Jones [11] have shown that $h_{n}(s) / h_{n}(1) \rightarrow H(s)$, the unique c.g.f. solution of

$$
H(h(s))=m H(s), \quad H(1)=1
$$

and having the canonical representation

$$
H(s)=\int_{0}^{\infty}\left(1-e^{-s x}\right) \Omega(d x)
$$

where $\Omega$ is a Lévy measure. In addition $\Omega((0, \infty))=\infty$ since $P_{1}\left(Z_{1}=0\right)=0[11$, page 219] and $\int_{0}^{\infty} x \Omega(d x)<\infty$ if and only if $E_{1}\left(Z_{1} \log ^{+} Z_{1}\right)<\infty$. The c.g.f. $H$ is that of the weak limit of the D.F.'s $P_{h_{n}^{-1}(1)}\left(Z_{n} \leqslant x\right)$ [11, page 215].

Using these results it is clear that the right hand side of (3.1) converges to

$$
\frac{H(s+\rho)-H(s)}{H(\rho)}=\frac{\int_{0}^{\infty} e^{-s x}\left(1-e^{-\rho x}\right) \Omega(d x)}{\int_{0}^{\infty}\left(1-e^{-\rho x}\right) \Omega(d x)}
$$

which is the Laplace-Stieltjes transform (L.S.T.) of a non-defective D.F. Consequently we have the following theorem.

TheOREM 3.1. If $r>0$ and $m<1$ then

$$
P_{z}\left(Z_{n} \leqslant x \mid \nu>n\right) \rightarrow G(x)=\frac{\int_{0}^{x}\left(1-e^{-\rho y}\right) \Omega(d y)}{\int_{0}^{\infty}\left(1-e^{-\rho y}\right) \Omega(d y)}
$$

and $\int_{0}^{\infty} x d G<\infty$ if and only if $E_{1}\left(Z_{1} \log ^{+} Z_{1}\right)<\infty$. 
A critical ( $m=1)$ analogue of Theorem 3.1 is given by

THEOREM 3.2. If $m=1, r>0$ and $\gamma=\frac{1}{2} \operatorname{var}_{1}\left(Z_{1}\right)<\infty$ then

$$
P_{z}\left(Z_{n} \leqslant n x \mid \nu>n\right) \rightarrow\left(1-e^{-x / \gamma}\right)^{+} \text {. }
$$

Proof. Seneta [10] has shown that

$$
\frac{1}{n}\left[\frac{1}{h_{n}(s)}-\frac{1}{s}\right]=\gamma+\varepsilon_{n}(s)
$$

where, for each $0<\delta<\infty, \sup _{0 \leqslant s \leqslant \delta} \varepsilon_{n}(s) \rightarrow 0(n \rightarrow \infty)$. (The factor $1 / n$ is missing in the statement of (3.4) in [10].) This result is also given by Kallenberg [4] under the extraneous condition $P_{1}\left(Z_{1}=0\right)>0$. Using (3.4) it is easily seen that

$$
\frac{h_{n}(\rho+s / n)-h_{n}(s / n)}{h_{n}(\rho)} \rightarrow(1+\gamma s)^{-1},
$$

the L.S.T. of the limiting D.F. of the assertion.

We now consider the distribution of $W_{n}=Z-r^{-n} Z_{n}$ given that $\nu>n$. In the next theorem we compute its conditional L.S.T. using the same idea as used in [8, Theorem 4.5] for the C.B. case.

TheOREM 3.3.

$$
E_{2}\left(e^{-s W_{n}} \mid \nu>n\right)=\frac{\exp \left[-z h_{n}\left(a\left(s r^{-n}\right)\right)\right]-\exp \left[-z h_{n}(\rho)\right]}{1-\exp \left[-z h_{n}(\rho)\right]}
$$

Proof. Since $W_{n}=0$ a.s on $\{\nu \leqslant n\}$ we have

$$
E_{z}\left(e^{-s W_{n}} ; \nu>n\right)=E_{z}\left(e^{-s W_{n}}\right)-P_{z}(\nu \leqslant n) .
$$

The first term is found by observing that

$$
\begin{aligned}
E\left(e^{\left.-s W_{n} \mid \mathscr{F}_{n}\right)}\right. & =\lim _{j \rightarrow \infty} E\left[\exp \left(-s\left(r^{-n-j} Z_{n+j}-r^{-n} Z_{n}\right)\right) \mid \mathscr{F}_{n}\right] \\
& =\lim _{j \rightarrow \infty} \exp \left[-Z_{n}\left(h_{j}\left(s^{-n-j}\right)-s r^{-n}\right)\right] \\
& =\exp \left[-Z_{n}\left(\gamma\left(s r^{-n}\right)-s r^{-n}\right)\right]=\exp \left(-Z_{n} a\left(s r^{-n}\right)\right)
\end{aligned}
$$

whence

$$
E_{z}\left(e^{-s W_{n}}\right)=\exp \left[-z h_{n}\left(a\left(s r^{-n}\right)\right)\right]
$$

and the assertion now follows upon using (2.1).

Since $h_{n}(\cdot) \rightarrow 0$ as $n \rightarrow \infty$ we obtain

$$
E_{z}\left[\exp \left(-s r^{n} W_{n}\right) \mid \nu>n\right] \sim \frac{h_{n}(\rho)-h_{n}(a(s))}{h_{n}(\rho)} .
$$

The next result gives a conditional limit theorem for $W_{n}$ in the subcritical case. 
Theorem 3.4. Suppose $0<r<m<1$. Then

$$
\lim _{n \rightarrow \infty} P_{z}\left(r^{-n} W_{n} \leqslant x \mid \nu>n\right)=\int_{0}^{\infty} P\left(C_{t} \leqslant x \mid C_{t}>0\right) G(d t)
$$

where $\left(C_{t}\right)$ is a compound Poisson process with c.g.f. $a(s)$ and $G$ is the limiting distribution in Theorem 3.1.

Proof. The results in [11] leading to (3.2) show that the left hand side of (3.5) converges to $1-H(a(s)) / H(\rho)$. Since $a(0)=0$ this is the L.S.T. of a non-defective D.F. and (3.3) enables us to represent this L.S.T. as $\int_{0}^{\infty} \phi(s, t) G(d t)$ where

$$
\phi(s, t)=\left[e^{-t a(s)}-e^{-\rho t}\right] /\left[1-e^{-\rho t}\right] .
$$

It was pointed out in [7] that (1.2) can be expressed as $r a(s)+k(s+a(s))=a(r s)$ and since $k(\infty)=n((0, \infty))=\lambda$, we obtain $a(\infty)=\rho$. Consequently $\exp (-t a(s))$ is the L.S.T. of a compound Poisson process $\left(C_{t}\right)$ with $C_{0}=0$, rate $\rho$ and jump D.F. $\rho^{-1} \mu((0, x])$ where $\mu$ is the Levy measure of $a(\cdot)$. It follows that $P\left(C_{t}=0\right)$ $=\exp (-\rho t)$ and hence that $\phi(s, t)$ is the L.S.T. of $P\left(C_{t} \leqslant x \mid C_{t}>0\right)$. This completes the proof.

Finally, we consider the critical case.

THEOREM 3.5. If $0<r<m=1$ and $\gamma=\frac{1}{2} \operatorname{var}_{1} Z_{1}<\infty$ then for $x>0$

$$
\lim _{n \rightarrow \infty} P_{z}\left(n a\left(r^{-n} W_{n}^{-1}\right) \leqslant x \mid \nu>n\right)=\frac{\gamma x}{1+\gamma x} \text {. }
$$

Proof. Equation (1.2) implies $\gamma(r s) / \gamma(s)=h(\gamma(s)) / \gamma(s) \rightarrow 1$ as $s \rightarrow 0$, and since $h$ is increasing it follows that $h$, and $a$, is S.V. at the origin. In (3.5) replace $s$ by $s a^{-1}(x / n)$, whence, from the slow variation of $a, a\left(s a^{-1}(x / n)\right) \sim x / n$. Using (3.4) we obtain

$$
E_{z}\left(\exp \left(-s r^{n} a^{-1}(x / n) W_{n}\right) \mid \nu>n\right) \rightarrow(1+\gamma x)^{-1} .
$$

The right hand side is the L.S.T. of a defective distribution which places mass $(1+\gamma x)^{-1}$ at the origin and no mass in $(0, \infty)$. It follows that

$$
P_{z}\left(r^{n} a^{-1}(x / n) W_{n} \leqslant 1 \mid \nu>n\right) \rightarrow(1+\gamma x)^{-1}
$$

and algebraic manipulation yields (3.6).

\section{The C.B. process}

A version of Theorem 1.1 for C.B. processes $\left(X_{t}\right)$ with $D \leqslant 0$ can be obtained from Theorem 1.1 by using the method of discrete skeletons [5] after we have checked a few conditions. First we need some notation. Let

$$
\pi(s)=\int_{0+}^{\infty}\left(1-e^{-s x}\right) \Pi(d x), \quad \psi(s)=-c s+\pi(s),
$$


assume $\Pi((0, \infty))=\infty$ and

$$
\int_{1}^{\infty} \pi(s) s^{-2} d x<\infty
$$

In this case [3] a.s. $e^{c t} X_{t} \rightarrow X<\infty$ whose c.g.f. $\eta$ satisfies the differential equation

$$
\eta^{\prime}(s) / \psi(\eta(s))=-1 / c s .
$$

Let $\psi_{t}(s)=-\log E_{1}\left(e^{-s X_{t}}\right)$, so $E_{z} e^{-s X_{t}}=\exp \left(-z \psi_{t}(s)\right)$. The c.g.f. $\psi_{t}$ satisfies

$$
\int_{s}^{\psi_{t}(s)} d u / \psi(u)=t
$$

Since $\psi(s) / s \rightarrow-c$ as $s \rightarrow \infty$ it follows from (4.3) that

$$
\lim _{s \rightarrow \infty} \psi_{t}(s) / s=e^{-c t} .
$$

Thus if $\left(X_{n}^{\tau}\right)$ is the discrete skeleton at scale $\tau>0$, that is, $X_{n}^{*}=X_{n \tau^{\prime}}$, then the first point of increase of the D.F. of $X_{1}^{\tau}$ is $r_{\tau}=e^{-c \tau}$. The a.s. convergence result cited above and that for the J.P. show that (4.1) is equivalent to

$$
\int_{1}^{\infty}\left(\psi_{t}(s)-s e^{-c t}\right) s^{-2} d s<\infty
$$

and that

$$
\lim _{s \rightarrow \infty} \eta(s) / s=1 \text {. }
$$

Let $D_{t}=W-e^{c t} X_{t}$. It follows from Theorem 1.1(i) that for each countable dense subset $\mathscr{D}$ of $\mathbb{R}_{+}$there is a set $\Delta$ such that $P_{z}(\Delta)=1$ and if $\omega \in \Delta$ then $D_{n \tau}(\omega) \geqslant 0$ for each $n \in N_{+}$and $\tau \in \mathscr{D}$. If necesary we can delete a set of zero probability from $\Delta$ so that for each $\omega \in \Delta, X$. $(\omega)$ is right-continuous and has left limits. Thus there is a sequence $\left(\tau_{i}\right) \subset \mathscr{D}$ such that $n \tau_{i} \downarrow t$ and $D_{n \tau_{i}}(\omega) \rightarrow D_{t}(\omega)$. We conclude that $P_{z}\left(D_{t} \geqslant 0, t \geqslant 0\right)=1$.

Suppose

$$
\pi(s)=s^{\delta} L(s) \quad(0 \leqslant \delta \leqslant 1)
$$

where $L$ is S.V. at infinity and when $s=1$ we must have $\int_{1}^{\infty} s^{-1} L(s) d s<\infty$ so that (4.1) is satisfied. The differential equation version of (4.3) can be solved in the form

$$
\psi_{t}(s)-s e^{-c t}=e^{-c t} \int_{0}^{t} e^{c u} \pi\left(\psi_{u}(s)\right) d u .
$$

When $\delta<1(4.4)$ and (4.6) yield

$$
\psi_{t}(s)-s e^{-c t} \sim \frac{s^{\delta} L(s)}{c(1-\delta)}\left(e^{-\delta c t}-e^{-c t}\right) \quad(s \rightarrow \infty)
$$

and when $\delta=1$,

$$
\psi_{t}(s)-s e^{-c t} \sim s L(s) t e^{-c t} \quad(s \rightarrow \infty) .
$$


Thus the conditions of Theorem 1.1 are satisfied for the skeleton $\left(X_{n}^{\tau}\right)$. Moreover it follows from [7] (Lemma 2 when $0 \leqslant \delta<1$ and equation (9) when $\delta=1$ ) that $a(s)=\eta(s)-s$ is R.V. with index $\delta$. This can also be deduced from (4.6) and (4.2). Consequently when $\delta>0$ the inverse $g$ of $a$ is $\delta^{-1}$-varying and the following result now follows from Theorem 1.1 and Kingman's Croftian theorems [5].

THeOREM 4.1. Let $D \leqslant 0,(4.1)$ and (4.6) hold for the C.B. process. When $0<\delta \leqslant 1$ then

$$
e^{c t} g\left(e^{c t}\right) D_{t} \stackrel{\mathscr{D}}{\rightarrow} S X^{1 / \delta}
$$

where $S$ is stable with c.g.f. $s^{\delta}$ and is independent of $X$. In addition

$$
e^{-c t} g\left(X_{t}^{-1}\right) D_{t} \stackrel{\mathscr{D}}{\rightarrow} S
$$

If $\delta=0$ and $L(s) \rightarrow \infty$ as $s \rightarrow \infty$ then

$$
\eta\left(e^{-c t} a\left(e^{c t} D_{t}^{-1}\right)\right) \stackrel{\mathscr{D}}{\rightarrow \Delta}
$$

where $P(\Delta>x)=e^{-x}$.

We now look at the special case of the supercritical C.B. process in which $c<0, \Pi((0, \infty)) \leqslant \infty$ and $\int_{0}^{\infty} x \Pi(d x) \leqslant \infty$ but $\left|\int_{0}^{\varepsilon} d s / \psi(s)\right|=\infty$. The last condition ensures that almost all sample paths are finite for all $t$ [3]. The condition $c<0$ ensures that almost all sample paths are non-decreasing and hence a.s. $X_{t} \uparrow \infty$ as $t \uparrow \infty$. Now consider a family of processes $\left(X_{t, u}: t \geqslant 0, u>0\right)$ defined by the same c.g.f. $\psi$ but with initial quantity of population $z / \psi_{u}(1)$. The following result asserts that the family $\left(X_{t, t}\right)$ converges in law. Since $\psi_{t}(1) \rightarrow \infty$, the population size is controlled by diluting the initial population size. Let $b=-c$.

THEOREM 4.2. The family $\left(X_{t, t}\right)$ has an infinitely divisible limiting distribution whose c.g.f. is $z Q(s)$ and $Q(s)$ solves the differential equation

$$
Q^{\prime}(s) / Q(s)=b / \psi(s) \text {. }
$$

If (4.1) holds, or equivalently if $\int_{0}^{1} x \log x^{-1} \Pi(d x)<\infty$, then we can replace $z / \psi_{u}(1)$ by $z e^{-b u}$. In this case $\lim _{s \rightarrow \infty} Q(s) / s=1$.

Proof. Theorem 6 in [7] implies that the skeletons $\left(X_{n \tau, n t}\right)$ have infinitely divisible limiting distributions whose c.g.f.'s have the form $z Q_{\tau}(s)$. Since the c.g.f. of $\left(X_{t, t}\right)$ is continuous in $t$, Kingman's theorem shows that $Q_{\tau}(\cdot)$ is independent of $\tau$ and $\left(X_{t, t}\right)$ converges in law to a distribution with c.g.f. $z Q(s)$. Pakes' result 
[7] asserts that $Q$ is, up to multiplicative constants, the unique solution of

$$
Q\left(\psi_{\tau}(s)\right)=e^{b \tau} Q(s)
$$

such that $Q(s) / s$ is monotone-recall that $e^{b \tau}$ is the first point of increase of the D.F. whose c.g.f. is $\psi_{\tau}(s)$. Differentiating (4.8) with respect to $\tau$ and using the backward equation $\partial \psi_{t} / \partial t=\psi\left(\psi_{t}\right)($ see (4.3)) gives (4.7).

Since (4.1) and (4.5) are equivalent, it follows from Pakes' theorem that (4.1) implies $\psi_{n \tau}(1) \sim$ const. $e^{n b \tau}(n \rightarrow \infty)$. Invoking Kingman's theorem once again implies that $\psi_{t}(1) \sim$ const. $e^{b t}$. The D.F. whose c.g.f. is $e^{-b t} \psi_{t}(s)$ has first point of increase equal to unity and, as can be inferred from (4.8), this is preserved in the limit, that is, $Q(s) / s \rightarrow 1$ as $s \rightarrow \infty$. This completes the proof.

Eq. (4.7) can be explicitly solved in a few cases. For example suppose $\Pi((x, \infty))=a e^{-\mu x}$ so that the generating subordinator $\left(L_{t}\right)$ is a compound Poisson process, with rate $a$ and exponential jump D.F. having mean $\mu^{-1}$, and with a superimposed linear drift with rate $b$. With an initial population size $z e^{b t}$, (4.7) becomes

$$
(\log Q(s))^{\prime}=\frac{b(\mu+s)}{s[a+b \mu+b s]}
$$

and this can be solved subject to the condition $Q(s) / s \rightarrow 1$. The solution is

$$
Q(s)=\left[s^{\mu}(\mu+\alpha+s)^{\alpha}\right]^{1 /(\mu+\alpha)}
$$

where $\alpha=\lambda / b$. The canonical representation of $Q$ is $s+\int_{0+}^{\infty}\left(1-e^{-s x}\right) \zeta(d x)$ where $\zeta$ is a Levvy measure and $\zeta((0, \infty)=\alpha$. If $\mu=\alpha$, then

$$
\alpha^{-1} \zeta((0, x])=\int_{0}^{x} y^{-1} e^{-\alpha y} I_{1}(\alpha y) d y
$$

where $I_{1}$ is the modified Bessel function.

\section{The residual integral}

We begin by considering the C.B. process with $c>0, D \leqslant 0$ and $a=$ $\Pi((0, \infty))<\infty$. In this case there is a last jump epoch, $T, X_{t+T}=X_{T} e^{-c t}$ and hence $\mathscr{I}_{R}=\int_{T}^{\infty} X_{t} d t=c^{-1} X_{T}$. Let $\phi(s)=1-a^{-1} \pi(s)$ be the L.S.T. of the jump size D.F. of $\left(L_{t}\right)$. We prove the following result about the size of the process at $T$. Let $\bar{\rho}=a / c$ which is analogous to $\rho$ as used in Sections 2 and 3 .

Theorem 5.1. When $D \leqslant 0$ and $a<\infty$ then

$$
E_{z}\left(e^{-s X_{T}} ; T>t\right)=\frac{-a \phi(\bar{\rho}+s)}{\psi(\bar{\rho}+s)}\left(1-e^{-z \psi_{t}(\bar{\rho}+s)}\right) .
$$


Moreover $\lim _{t \rightarrow \infty} P_{z}\left(X_{T} \leqslant x \mid T>t\right)=A(x)$ exists as a proper non-degenerate D.F. and

$$
\int_{0}^{\infty} e^{-s x} d A(x)=\frac{-a \phi(\bar{\rho}+s)}{\psi(\bar{\rho}+s)} \cdot \frac{\chi(\bar{\rho}+s)}{\chi(\bar{\rho})}
$$

where $\chi(s)=\lim _{t \rightarrow \infty} \psi_{t}(s) / \psi_{t}(1)$. When $D<0$ then $\chi$ is the c.g.f. given by

$$
\chi(s)=\exp \left(D \int_{1}^{s} d u / \psi(u)\right)
$$

and when $D=0$ then $\chi(s) \equiv 1$.

Proof. Given $\mathscr{F}_{t}$, a jump of size $Y$ occurs in $(t, t+d t)$ with probability $a X_{t} d t+o(d t)$ and no jumps occur after $t+d t$ with probability $e^{-\bar{\rho}\left(X_{t}+Y\right)}+o(d t)$. Thus, excluding terms of order $o(d t)$, we have

$$
\begin{aligned}
E_{z}\left(e^{-s X_{T}} ; t<T<t+d t\right) & =E_{z}\left(e^{-\left(X_{t}+Y\right)(s+\bar{\rho})} a X_{t} d t\right) \\
& =a \phi(\bar{\rho}+s)\left(-\partial / \partial s e^{-z \psi_{t}(\bar{\rho}+s)}\right) d t \\
& =a z \phi(\bar{\rho}+s)\left(\frac{\partial \psi_{t}(\bar{\rho}+s)}{\partial s}\right) e^{-z \psi_{t}(\bar{\rho}+s)} d t \\
& =\frac{a z \phi(\bar{\rho}+s)}{\psi(\bar{\rho}+s)}\left(\frac{\partial \psi_{t}(\bar{\rho}+s)}{\partial t}\right) e^{-z \psi_{t}(\bar{\rho}+s)} d t
\end{aligned}
$$

and we have used the forward equation. Integration now yields (5.1)-recall that $\psi_{t}(s) \rightarrow 0$ as $t \rightarrow \infty$. In addition, as $t \rightarrow \infty$

$$
E_{z}\left(e^{-s X_{T}} \mid T>t\right) \sim \frac{a \phi(\bar{\rho}+s)}{\psi(\bar{\rho}+s)} \cdot \frac{\psi_{t}(\bar{\rho}+s)}{\psi_{t}(\bar{\rho})} .
$$

When $D<0$ it was shown in [8] that $\psi_{t}(s) / \psi_{t}(1) \rightarrow \chi(s)$ as given by (5.3). In [8] $h(\cdot)$ is used instead of $\chi(\cdot)$-see equation (4.6) therein.

For the case $D=0$ let $0<s_{1}<s_{2}$. Since $\psi(s)<0$ it follows from (4.3) that $\psi_{t}\left(s_{1}\right)<\psi_{t}\left(s_{2}\right)$. Now $\varepsilon(s)=\psi(s) / s \rightarrow 0$ as $s \rightarrow 0$, so using (4.3) again we have

$$
\int_{s_{1}}^{s_{2}} d u / \psi(u)=\int_{\psi_{t}\left(s_{1}\right)}^{\psi_{t}\left(s_{2}\right)} d u / u \varepsilon(u)=\left(\varepsilon\left(\zeta_{t}\right)\right)^{-1} \log \frac{\psi_{t}\left(s_{2}\right)}{\psi_{t}\left(s_{1}\right)}
$$

where $\psi_{t}\left(s_{1}\right)<\zeta_{t}<\psi_{t}\left(s_{2}\right)$. Since the left hand side is independent of $t$ we must have $\psi_{t}\left(s_{2}\right) / \psi_{t}\left(s_{1}\right) \rightarrow 1$ as $t \rightarrow \infty$.

It follows that when $D \leqslant 0$ the right hand side of (5.4) converges to that of (5.2) with $\chi$ as described in the assertion. Since $\psi(\bar{\rho})=-a \phi(\bar{\rho})$, the right hand side of (5.2) tends to unity as $s \rightarrow 0$ and hence is the L.S.T. of a proper non-degenerate D.F. The proof is complete. 
Note the explicit form of the limiting L.S.T. when $D=0$. As an example let $\phi(s)=\mu /(\mu+s)$ and $c=a / \mu$, ensuring $D=0$. The L.S.T. at (5.2) is then $[\bar{\rho} /(\bar{\rho}+s)]^{2}$ and hence the limiting distribution is a scaled $\chi_{4}^{2}$-distribution.

We now obtain a similar result for the J.P. but here the limiting L.S.T.'s involve functions that satisfy functional equations.

THEOREM 5.2. Let $0<r<m \leqslant 1$. Then

$$
E_{z}\left(e^{-s Z_{\nu}} ; \nu>n\right)=\sum_{j=0}^{\infty}\left\{\exp \left(-z h_{n+j+1}(\rho+s)\right)-\exp \left(-z h_{n+j}(\rho+r s)\right)\right\} .
$$

and as $n \rightarrow \infty$

$$
P_{z}\left(Z_{v} \leqslant x \mid \nu>n\right) \rightarrow B(x),
$$

a proper non-degenerate D.F.

When $m<1$

$$
\int_{0}^{\infty} e^{-s x} d B(x)=\frac{H(\rho+r s)-m H(\rho+s)}{(1-m) H(\rho)}
$$

where $H$ is defined in Section 3.

When $m=1$

$$
\int_{0}^{\infty} e^{-s x} d B(x)=\beta(s)=1+V(r s)-V(s)
$$

where $V(s)$ is the c.g.f. defined by

$$
V(s)=\lim _{n \rightarrow \infty} \frac{h_{n}(\rho+s)-h_{n}(\rho)}{h_{n}(\rho)-h_{n+1}(\rho)}
$$

and satisfying the functional equation

$$
V(h(s)-\rho)=V(s-\rho)-1 .
$$

Proof. The left hand side of (5.5) is

$$
\sum_{i=n+1}^{\infty} E_{z}\left(e^{-s Z_{i}} ; \nu=i\right)=\sum_{i=n+1}^{\infty}\left[E_{z}\left(e^{-s Z_{i}} ; \nu>i-1\right)-E_{z}\left(e^{-s Z_{i}} ; \nu>i\right)\right] \text {. }
$$

The second term in the summand is given by equation (2.1) and the first term is $E_{z}\left(e^{-s Z_{i}}\right)-E_{z}\left(e^{-s Z_{i}} ; \nu \leqslant i-1\right)$. When $\nu \leqslant i-1, Z_{i}=r Z_{i-1}$ and hence Theorem 2.1 yields $E_{z}\left(e^{-s Z_{i}} ; \nu \leqslant i-1\right)=\exp \left(-z h_{i-1}(\rho+r s)\right)$. Putting these expressions together gives (5.5) and using Theorem 2.1 again we obtain

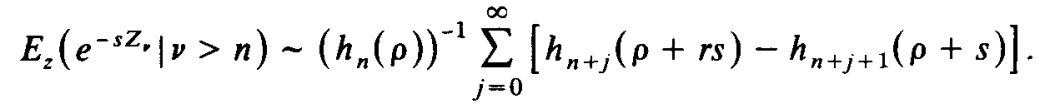


When $m<1$ the definition of $H(\cdot)$ shows that

$$
\lim _{n \rightarrow \infty} E_{z}\left(e^{-s Z_{v}} \mid \nu>n\right)=(H(\rho))^{-1} \sum_{j=0}^{\infty}\left[H\left(h_{j}(\rho+r s)\right)-H\left(h_{j+1}(\rho+s)\right)\right] .
$$

However (3.2) can be iterated to yield $H\left(h_{j}(s)\right)=m^{j} H(s)$ and the right hand side of (5.11) sums to that of (5.7), which we denote by $\alpha(s)$. Since $\alpha(0+)=1$ the continuity theorem for L.S.T.'s yields (5.6) and (5.7).

When $m=1$ the conditions of the Lévy-Szekeres theorem [6, Chapter 7] are satisfied by $h(s)$ and it follows that

$$
v(s)=\lim _{n \rightarrow \infty}\left(h_{n}(s)-h_{n}(\rho)\right) /\left(\left(h_{n}(\rho)-h_{n+1}(\rho)\right)\right.
$$

exists, is continuous and strictly increasing, and is the unique convex solution of the Abel-type equation $v(h(s))=v(s)-1$. Defining $V(s)=v(\rho+s)$ gives (5.9). If $\Lambda_{n}$ is the Lévy measure of $h_{n}(s)$ then

$$
h_{n}(\rho+s)-h_{n}(\rho)=r^{n} s+\int_{0+}^{\infty}\left(1-e^{-s x}\right) d_{x} \int_{0}^{x} e^{-\rho y} \Lambda_{n}(d y)
$$

is a c.g.f. - note that $\Lambda_{n}((0, \infty))<\infty$. It follows [11, Appendix] that $V(s)$ is a c.g.f. having the canonical form $\int_{0+}^{\infty}\left(1-e^{-s x}\right) \Lambda(d x)$ where $\Lambda$ is the weak limit of the measures $\left(h_{n}(\rho)-h_{n+1}(\rho)\right)^{-1} \int_{A} e^{-\rho y} \Lambda_{n}(d y)$.

Let

$$
V_{n}(s)=\frac{h_{n}(\rho+s)-h_{n}(\rho)}{h_{n}(\rho)-h_{n+1}(\rho)} .
$$

The summands in (5.10) can be written as

$$
\left\{V_{n+j}(r s)-\frac{h_{n+j+1}(\rho)-h_{n+j+2}(\rho)}{h_{n+j}(\rho)-h_{n+j+1}(\rho)} V_{n+j+1}(s)+1\right\}\left(h_{n+j}(\rho)-h_{n+j+1}(\rho)\right) \text {. }
$$

The Lévy-Szekeres theorem also states that the fraction term in the braces tends to unity, so for large enough $n$ and all $j$ the term in braces is arbitrarily close to $\beta(s)$. Thus

$$
E_{z}\left(e^{-s Z_{\nu}} \mid \nu>n\right) \sim \beta(s)\left(h_{n}(\rho)\right)^{-1} \sum_{j=0}^{\infty}\left(h_{n+j}(\rho)-h_{n+j+1}(\rho)\right)=\beta(s) .
$$

Since $\beta(0)=1,(5.6)$ and (5.8) follow.

Now let $\left(U_{n}\right)$ be a Galton-Watson process with a non-linear offspring distribution p.g.f. $f(\theta)$ satisfying $M=f^{\prime}(1-) \leqslant 1$. Let $f_{n}(\theta)$ be the $n$-fold iterate of $f$ with $f_{0}(\theta)=\theta, f_{n}=f_{n}(0)$ and let $N=\min \left\{n: U_{n}=0\right\}$, the extinction time of $\left(U_{n}\right)$. A quantity analogous to the conditional distributions of Theorems 5.1 and 5.2 is $P_{i}\left(U_{N-1}=j \mid N>n\right)$. The following results correspond to these theorems. 
THEOREM 5.3. When $M \leqslant 1$

$$
E_{i}\left(\theta^{U_{N-1}} \mid N>n\right)=\left(1-f_{n}^{i}\right)^{-1} \sum_{j=0}^{\infty}\left[\left(f_{n+j}\left(f_{1} \theta\right)\right)^{i}-f_{n+j}^{i}\right] .
$$

In addition

$$
\lim _{n \rightarrow \infty} P_{i}\left(U_{N-1}=j \mid N>n\right)=p_{j}
$$

where $\left(p_{j}\right)$ is a distribution concentrated on $\mathbb{N}$.

When $M<1$ then

$$
\sum_{j=1}^{\infty} p_{j} \theta^{j}=\frac{Q\left(f_{1} \theta\right)}{1-M}
$$

where $Q$ is the p.g.f. defined by

$$
Q(\theta)=\lim _{n \rightarrow \infty} E\left(\theta^{U_{n}} \mid N>n\right)=\lim _{n \rightarrow \infty} \frac{f_{n}(\theta)-f_{n}}{1-f_{n}} .
$$

and satisfies the functional equation

$$
1-Q(f(\theta))=M(1-Q(\theta)) .
$$

When $M=1$ then

$$
\sum_{j=1}^{\infty} p_{j} \theta^{j}=U\left(f_{1} \theta\right)
$$

where

$$
U(\theta)=\lim _{n \rightarrow \infty} \frac{f_{n}(\theta)-f_{n}}{f_{n+1}-f_{n}}
$$

and satisfies the functional equation

$$
U(f(\theta))=1+U(\theta) .
$$

The proof is quite similar to that of Theorem 5.2 and uses the assertions about $Q$ and $U$ which can be found in [1] and [9], respectively. The interest here is that the limiting distribution has the form $p_{j}=$ const. $u_{j} f_{j}^{j}$ where $\left(u_{j}\right)$ is the $M^{-1}$-invariant measure of the matrix $\left[P_{i j}: i, j \geqslant 1\right]$ where $P_{i j}=P_{i}\left(U_{1}=j\right)$, that is,

$$
M^{-1} \sum_{i \leqslant 1} u_{i} P_{i j}=u_{j} \quad(j \geqslant 1) .
$$

When $M<1$ the $u_{j}$ can be taken $\lim _{n \rightarrow \infty} P_{1}\left(U_{n}=j \mid N>n\right)$ and (5.12) can be written as $Q(f(\theta))=M Q(\theta)+Q\left(f_{1}\right)$ which should be compared with equation 1.7.6. in [1]. When $M=1,\left(u_{j}\right)$ is the invariant measure of $\left(U_{n}\right)$ and $\sum u_{j}=\infty$. 
Connections like these seem not to exist for the continuous-state processes. In fact if $R \geqslant 1$ our processes do not have $R$-invariant measures which possess L.S.T.'s. For example suppose $\delta(\cdot)$ is such a measure for the J.P. and let $K(z, A)$ denote its one-step transition kernel. The measure $K(z, \cdot)$ is supported in $[r z, \infty)$. If $\Delta(s)=\int_{0+}^{\infty} e^{-s x} \delta(d x)$ exists then

$$
\Delta(s)=R \int_{0+}^{\infty} \int_{0+}^{\infty} \delta(d z) K(z, d x) e^{-s x}=R \Delta(h(s)) .
$$

Iterating this equation gives

$$
R^{-n} \Delta(s)=\Delta\left(h_{n}(s)\right) .
$$

If $R=1$ then $\Delta(\cdot)$ must be constant, and if $R>1$ then the left hand side decreases as $n$ increases but the right hand side increases. It seems likely that non-trivial $R$-invariant measures will exist only for those continuous-state branching processes which can hit the origin with positive probability.

\section{Acknowledgement}

The research described here was carried out while the author enjoyed the hospitality of Colorado State University.

\section{References}

[1] K. B. Athreya and P. E. Ney, Branching processes (Springer-Verlag, Berlin, 1972).

[2] L. Breiman, Probability (Addision-Wesley, Reading, Mass., 1968).

[3] D. R. Grey, 'Asymptotic behavior of continuous time, continuous state-space branching processes', J. Appl. Probab. 11 (1974), 669-677

[4] P. J. M. Kallenberg, Branching processes with continuous state space (Math. Centrum, Amsterdam, 1979).

[5] J. F. C. Kingman, 'Ergodic properties of continuous-time Markov processes and their discrete skeletons', Proc. London Math. Soc. 13 (1963), 593-604.

[6] M. Kuczma, Functional equations in a single variable (PWN, Warsaw, 1968).

[7] A. G. Pakes, 'Some limit theorems for Jiřina process', Period. Math. Hungar. 10 (1979), 55-66.

[8] A. G. Pakes and A. C. Trajstman, 'Some properties of continuous-state branching processes, with applications to Bartoszynski's virus model', Adv. in Appl. Probab. 17 (1985), 23-41.

[9] E. Seneta, 'The Galton-Watson process with mean one', J. Appl. Probab. 4 1967), 489-495. 
[10] E. Seneta, 'Some supplementary notes on one-type continuous-state branching processes', $Z$. Wahrsch. Verw. Gebiete 34 (1976), 87-89.

[11] E. Seneta and D. Vere-Jones, 'On the asymptotic behavior of subcritical branching processes with continuous state space', Z. Wahrsch. Verw. Gebiete 10 (1968), 212-225.

[12] E. Seneta and D. Vere-Jones, ' On a problem of M. Jifina concerning continuous state branching processes', Czech. Math. J. 19 (94) (1969), 277-283.

Department of Mathematics

University of Western Australia

Nedlands, 6009

Western Australia

Australia 